\title{
Un dossier de sources historiographiques et diplomatiques auxerroises : nouvelles éditions critiques
}

Thomas Granier

\section{(2) OpenEdition \\ Journals}

Édition électronique

URL : https://journals.openedition.org/cem/13359

DOI : 10.4000/cem.13359

ISSN : 1954-3093

Éditeur

Centre d'études médiévales Saint-Germain d'Auxerre

Référence électronique

Thomas Granier, « Un dossier de sources historiographiques et diplomatiques auxerroises : nouvelles éditions critiques ", Bulletin du centre d'études médiévales d'Auxerre | BUCEMA [En ligne], 18.1 | 2014, mis en ligne le 18 juin 2014, consulté le 22 septembre 2022. URL : http://journals.openedition.org/cem/ 13359 ; DOI : https://doi.org/10.4000/cem.13359

Ce document a été généré automatiquement le 22 septembre 2022.

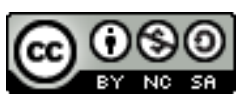

Creative Commons - Attribution - Pas d'Utilisation Commerciale - Partage dans les Mêmes Conditions 4.0 International - CC BY-NC-SA 4.0

https://creativecommons.org/licenses/by-nc-sa/4.0/ 


\title{
Un dossier de sources
}

\section{historiographiques et diplomatiques auxerroises : nouvelles éditions critiques}

\author{
Thomas Granier
}

1 Entre 2002 et 2012 sont parues plusieurs éditions critiques de textes historiographiques et diplomatiques auxerrois des $\mathrm{IX}^{\mathrm{e}}$-XIII ${ }^{\mathrm{e}}$ siècles. L'accès désormais facilité à ces sources est déjà à lui seul à saluer; surtout, leur confrontation maintenant possible permet des éclairages nouveaux sur l'histoire d'Auxerre au Moyen Âge.

2 Les Gestes des évêques d'Auxerre. Sous la

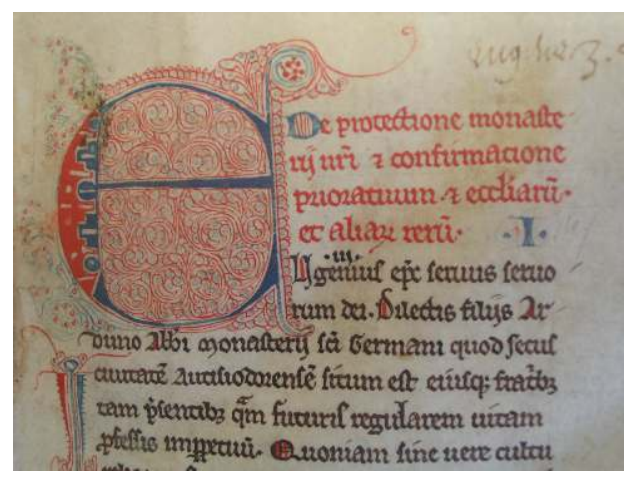
direction de Michel Sot (Les classiques de l'histoire [de France] au Moyen Âge, 42, 43 et 47), 3 tomes, 19 cm, LVII +336 p., 372 p. et XLI + 297 p., Paris, 2002-2009; ISBN 978-2-251-34053-1, 978-2-251-34054-8 et 978-2-251-34058-6; $41 €, 41 €$ et $43 €$ [le tome 2 ne distingue pas introduction et texte par une double numérotation].

3 Les Gestes des abbés de Saint-Germain d'Auxerre. Édition et traduction par Noëlle DeflouLeca et Yves Sassier (Les classiques de l'histoire au Moyen Âge, 50), 19 cm, LXII + 178 p., Paris, 2011 ; ISBN 978-2-251-34061-6, $43 €$.

4 Three Cartularies from Thirteenth-Century Auxerre. Edited by Constance Brittain Bouchard (Medieval Academy Books, 113), 23 cm, 259 p., Toronto/Buffalo/London 2012 ; ISBN 978-1-4426-4528-8. 
5 La publication des trois volumes des Gestes des évêques d'Auxerre est le fruit d'une entreprise collective à long terme, qui a réuni à partir de 1995 une équipe de dix chercheurs autour de Michel Sot. Il faut dès l'abord souligner que, si le texte paraît dans les Classiques de l'histoire au Moyen Âge, cette équipe a fait le choix d'éditer le texte dans son intégralité, avec toutes ses continuations successives jusqu'à la notice finale, celle de Nicolas Colbert - frère du Grand Colbert, †1676 -, rédigée en 1773. La publication en trois volumes ( 1100 pages) scinde le texte en trois parties : des origines à la fin du XI ${ }^{\mathrm{e}}$ siècle, 1092-1278, et de la fin du XIII à la fin du XVII ${ }^{\mathrm{e}}$ siècle. Les éditeurs respectent les normes de la collection dans laquelle parait leur travail, avec la présentation du manuscrit et de l'œuvre, des auteurs et des circonstances de rédaction, des éditions précédentes et de la bibliographie, l'édition scientifique du texte et sa traduction française en doubles pages affrontées et les identifications précises des sources, des personnages et des lieux ; ils y ajoutent deux originalités très bienvenues : une sérieuse étude de l'œuvre du point de vue littéraire et du genre (t. 1, p. XXI-XXXIV et t. 3, p. XXVII-XXXIv) et un essai d'histoire globale de l'église d'Auxerre, largement mais pas seulement appuyé sur les Gestes, distribué entre les trois volumes (t. 1, p. XII-XX ; t. 2, p. 14-40 et t. III, p. XII-XXVI) ; cet essai, qui permet de bien mettre en perspective le texte lui-même et sa teneur, s'accompagne de tableaux chronologiques précis de la série épiscopale (t. 1, p. LIV-LVII, t. 2, p. 44-45 et t. 3, p. XxXVI-XLI), qui proposent de très utiles corrections aux chronologies précédentes.

6 La très longue poursuite, sur presque exactement neuf cents ans, du processus de rédaction fait la particularité des Gestes auxerrois. Le texte est entièrement rédigé dans le milieu des chanoines cathédraux, en latin jusqu'à la fin. Tous les évêques connus jusqu'en 1676 font l'objet d'une notice, sauf pour les années 1372-1513 et 1554-1570. L'intérêt est exclusivement centré sur Auxerre, sauf lorsqu'un évêque participe à des événements importants à plus grande échelle, que ce soit Germain (418-448) ou Guy de Mello (1247-1270), parti en Italie méridionale avec Charles d'Anjou. À partir du XIV ${ }^{e}$ siècle, Auxerre n'est souvent qu'une étape dans une carrière épiscopale, et le texte ne détaille alors pas la suite de la vie des évêques après leur transfert. Comme c'est le cas le plus fréquent pour les œuvres de ce genre, les Gestes sont transmis par un manuscrit unique, aujourd'hui conservé en deux parties démembrées: AUXERRE, Bibliothèque municipale, 142 et CITTÀ DEL VATICANO, Biblioteca Apostolica Vaticana, Reg. lat. 1283A. Les p. 1-221 du manuscrit 142 sont une copie d'un seul trait, du milieu du XII ${ }^{\text {e }}$ siècle, de l'ensemble des notices jusqu'à celle d'Hugues de Mâcon (1136-1151), lissant donc les différentes étapes de rédaction jusque-là, que les éditeurs s'efforcent de restituer, discutant et corrigeant si nécessaire et si possible les propositions faites par Pierre Janin dans sa thèse de l'École des chartes de 1969 (t. 1, p. VIII-XII). Tout commence par un premier noyau, couvrant la période des origines de l'Église d'Auxerre à l'évêque Christianus ( $† 872)$, rédigé vers $873-875$, soit exactement en même temps que les Miracles de Germain par le moine Heiric de Saint-Germain ; chanoines et abbaye ont des objectifs et des intérêts très différents et choisissent des genres textuels bien distincts ; les deux entreprises sont néanmoins liées, chacun des deux textes renvoyant à l'autre (t. 1, p. xI). Ce noyau d'origine et ses diverses continuations, du $x^{e}$ au xVIII siècle, respectent l'organisation, caractéristique du genre, en notices successives consacrées à chaque évêque, selon une trame commune - naissance, personnalité, carrière, principales actions, fondations et constructions, legs à l'église, mort, sépulture et récapitulation chronologique - inspirée du Liber pontificalis romain, dont il existe 
d'ailleurs un manuscrit auxerrois du deuxième quart du IX siècle, utilisé par les rédacteurs : le canal de l'influence du modèle romain sur l'entreprise des Gestes est ici matériellement identifié.

Sans retracer ici dans tous ses détails le processus de composition, on peut faire remarquer que plusieurs continuations sont rédigées à des moments importants dans l'histoire de l'évêché :

- la notice de Gualdric († 933) s'achève sur la mention de l'élévation des reliques des évêques Pallade (623-659) et Trétice (692-707) durant la onzième année de Guy, son successeur (vers 944), mention assortie d'une récapitulation chronologique, qui permet de penser à une première continuation (pour la période 872-933) rédigée à cette occasion : l'intérêt pour les reliques de deux saints prédécesseurs coïncide avec un regain d'intérêt pour l'histoire des évêques (t. 1, p. 220-221);

- l'évêque Geoffroy (1054-1076) fait renovare les Gestes en même temps qu'il restaure le trésor liturgique de la cathédrale : là encore, une étape importante de l'histoire du texte coïncide avec une restauration ou réorganisation de l'institution (t. 1, p. 266-267);

- Nicolas Colbert fait commencer une collection des statuts synodaux auxerrois depuis les plus anciens connus; cette entreprise reste inachevée et sa notice est d'un siècle postérieure. Il est donc difficile d'affirmer que l'activité administrative de l'évêque est la vraie raison de l'intérêt de l'auteur, pas plus que l'on ne peut expliquer pourquoi il s'arrête un siècle avant sa propre époque. Cette dernière notice reste sans suite : trait commun du genre, là encore, les Gestes auxerrois ne sont ni achevés ni inachevés; vient un moment (exceptionnellement tardif ici) où l'on cesse de continuer, sans que l'on puisse forcément expliquer pourquoi.

8 Le degré d'information des auteurs sur leurs personnages est extrêmement variable. Parmi les évêques anciens, seuls Amâtre ( $† 418)$ et Germain, c'est-à-dire ceux pour qui existent des traditions hagiographiques, font l'objet de notices détaillées. Germain est d'ailleurs le seul évêque précisément et correctement daté jusqu'à Aunaire (561-604), qui est aussi celui avec lequel apparaissent les documents insérés dans les notices : à part le cas exceptionnel de Germain, c'est donc vers 600 que semblent apparaître à la fois documents d'archives et information chronologique fiable. Sur les évêques des XII ${ }^{\mathrm{e}}$ $\mathrm{XIII}^{\mathrm{e}}$ siècles, l'information est très inégale, y compris lorsque les auteurs sont de peu postérieurs aux faits. Les notices des deux évêques des années 1184-1220, rédigées ensemble peu après 1223 , sont de très loin les plus longues et détaillées de toute l'œuvre ; à l'inverse, d'autres sont extrêmement courtes, trois se réduisant à une ou deux doubles pages dans l'édition. La partie du texte conservée dans le manuscrit du Vatican est une unité cohérente, correspondant à une étape de rédaction: après environ un siècle d'interruption - la dernière notice du manuscrit 142 est celle d'Érard, $† 1277$-, l'évêque Nicolas d'Arcy (1372-1376) charge un chanoine de reprendre le récit ; celui-ci s'exécute, avec une information très inégale: il en sait peu sur les évêques anciens, de la fin du XIII et du début du XIV siècle, et bien plus sur les plus récents, en particulier les quatre derniers, dont il a été le collaborateur ; il expose les circonstances de son travail dans un long Avertissement (t. 3, p. 6-11).

9 L'édition des Gestes des abbés de Saint-Germain est le fruit du travail mené par Noëlle Deflou-Leca en relation avec la réalisation de sa thèse, Saint-Germain d'Auxerre et ses dépendances, $V^{e}$-XIII ${ }^{e}$ siècle. Un monastère dans la société du haut Moyen Âge (CERCOR), SaintÉtienne, 2010. Ce texte est lui aussi transmis par un témoin unique: PARIS, BnF, lat. 10940. Son auteur, l'abbé Gui (1285-1309), conçoit toute l'organisation de l'œuvre en fonction des rapports de son monastère avec Cluny : fait assez exceptionnel de ce genre 
de textes, il reconnaitt la grande ancienneté de son institution ( $v^{e}$ siècle), mais passe ses origines sous silence, déclarant tout ignorer de son histoire jusqu'à la première réforme clunisienne de Saint-Germain, sous l'abbé Heldric (989-1010). Une seconde, sous l'abbé Hugues de Montaigu (v. 1099-1115), n'entraîne pas la réduction de l'abbaye au rang de prieuré. Gui achève son travail vers 1290 , justement alors que Saint-Germain connaît une décisive émancipation vis-à-vis de Cluny; la compilation, par Gui lui-même, du cartulaire de l'abbaye un peu plus tôt est elle aussi à relier à cette étape majeure.

10 À la fin du XIII siècle, Gui recourt à un genre déjà fort ancien, plus guère usité depuis le XII ${ }^{e}$ siècle, sauf à Auxerre justement : son travail s'inspire des Gestes des évêques - dans sa préface, il évoque des gesta pontificum Romanorum et Autissiodorensium (p. 6) - et est de peu postérieur à la rédaction de la notice de l'évêque Érard de Lésignes, peu après 1278. Ce travail est continué deux fois : sa propre notice est rédigée peu après 1314, puis celle de son successeur Gaucher (1314-1335) peu après 1335. La fin du manuscrit porte, de différentes mains modernes, quelques brèves mentions sur certains abbés postérieurs, jusqu'à Étienne-Charles Loménie de Brienne $(\dagger 1720)$. Gui et ses deux continuateurs travaillent de deux façons fort différentes : l'abbé écrit surtout de l'histoire ancienne, sur laquelle il n'a quasiment aucun témoignage narratif ; il s'appuie donc avant tout sur la documentation d'archives, qu'il connaît particulièrement bien puisqu'il l'a lui-même classée. Le processus de réalisation du cartulaire est d'ailleurs évoqué dans sa propre notice (p. 108-111) et, chaque fois que c'est possible, l'éditrice identifie de façon précise en note les références des Gestes au Grand Cartulaire de Saint-Germain (AUXERRE, BM, ms. 161G). Gui, dans sa dernière notice, consacrée à l'abbatiat de son prédécesseur, et ses deux continuateurs, écrivent en revanche de l'histoire contemporaine, rapportant des faits dont ils ont été directement témoins.

11 Grâce à ces quatre volumes de Gestes auxerrois, qui viennent s'ajouter à l'édition par Pascal Pradié de la Chronique des abbés de Fontenelle du milieu du IX ${ }^{\mathrm{e}}$ siècle (Les classiques de l'histoire de France au Moyen Âge, 40, Paris, 1999), on dispose désormais de quelques témoins aisément accessibles et manipulables de cet important genre historiographique.

L'ouvrage de Constance Bouchard prolonge ses deux livres précédents, Spirituality and Administration: The Role of the Bishop in Twelfth-Century Auxerre, 1979, et Sword, Miter and Cloister: Nobility and the Church in Burgundy, 980-1198, 1987, et s'ajoute à ses éditions des cartulaires de Flavigny, Saint-Marcel-lès-Chalon et Montier-en-Der, parues respectivement en 1991, 1998 et 2004. Sont ici concernés trois cartulaires jusqu'ici inédits, dont les pièces n'étaient pas forcément toutes connues :

\begin{tabular}{|l|l|l|l|l|}
\hline Désignation & Référence & Date & $\begin{array}{l}\text { Nombre de } \\
\text { pièces }\end{array}$ & $\begin{array}{l}\text { Période } \\
\text { couverte }\end{array}$ \\
\hline $\begin{array}{l}\text { Cartulaire du chapitre } \\
\text { cathédral }\end{array}$ & Auxerre, AD Yonne G1797 & $\begin{array}{l}\text { années } \\
1230\end{array}$ & 11 & $1166-1230$ \\
\hline Cartulaire de l'évêché & $\begin{array}{l}\text { Berkeley, University of } \\
\text { California, ms Robbins MS48 }\end{array}$ & $\begin{array}{l}\text { années } \\
1280\end{array}$ & 103 & $1142-1281$ \\
\hline $\begin{array}{l}\text { Cartulaire du monastère } \\
\text { Saint-Julien }\end{array}$ & Auxerre, AD Yonne H1667 & $\begin{array}{l}\text { années } \\
1290\end{array}$ & 14 & $631-1291$ \\
\hline
\end{tabular}


13 Le cartulaire de l'évêché, l'un des rares documents des archives épiscopales auxerroises à avoir échappé à l'incendie de la résidence des évêques à Régennes (commune d'Appoigny, Yonne) en 1569, considéré comme détruit depuis la Révolution et retrouvé au milieu du $\mathrm{xx}^{\mathrm{e}}$ siècle, est aujourd'hui conservé par la Law School de l'université de Berkeley (Californie). Il laisse en particulier entrevoir la réalité matérielle du classement des archives épiscopales à la fin du xiII ${ }^{e}$ siècle. Le compilateur déclare que les originaux sont conservés dans une série de coffres (scrinia) marqués des lettres de l'alphabet $\mathrm{A}$ à $\mathrm{T}$ - le J est alors inexistant -, chaque coffre correspondant à un type de droits ou à un lieu ou institution concerné, et transcrire selon l'ordre de ces coffres : Hic incipit liber cartarum episcopi Autissiodorensis que inuenientur in scriniis signatis per ordinem alphabeti, et primo continentur littere que faciunt ad ius et iuridictionem episcopi in ciuitate Autissiodorensis et pertinentiis in scriniis signatis per A (p. 21); in scrinio signato per C inuenientur littere de homagio comitum et baronum (p. 51), etc. Le coffre B renferme les pièces relatives aux conflits sur la coutume du portage de l'évêque par ses principaux vassaux lors de son intronisation (p. 38), un rituel qui n'est en fait effectivement attesté par les Gestes que pour Pierre de Mortemart en 1325 (t. 3, p. 22-23). Le compilateur saute le coffre $\mathrm{R}$ - négligence ? Est-il momentanément indisponible? - et ne donne qu'un aperçu du contenu des coffres $\mathrm{S}$ et $\mathrm{T}$ - « ayant semble-t-il perdu tout entrain », commente l'éditrice p. 6 . Au XIV siècle, une liste de documents d'archives de l'évêché est ajoutée au cartulaire - fol. 8r-11v, ici p. 227-232 -, classée elle aussi selon ces coffres ainsi marqués. Les documents du cartulaire et de cette liste ne se recoupent qu'en partie, ce qui témoigne certes du caractère évolutif du corpus d'archives, mais plus encore des choix de transcription du scribe: lassitude peut-être vers la fin, mais surtout sélection raisonnée, puisqu'il ne transcrit en général pas de documents similaires ou répétitifs, préférant en copier un et signaler les autres - par exemple la série de onze Item alia littera ou Item littera du coffre C, p. 54-55. Trois (seulement) des cent trois actes $\mathrm{du}$ cartulaire sont transmis par ailleurs, ce qui permet une comparaison: les bonnes correspondances entre les versions permettent de conclure que le compilateur transcrit rigoureusement les documents dont il dispose.

Les deux actes les plus anciens, la confirmation des droits de l'évêché sur SaintGermain par Innocent II en 1142 - n 96 , dans le coffre $O$ - et la confirmation par Bernard de Clairvaux d'un compromis entre l'évêque et le comte de Nevers en 1145 $\mathrm{n}^{\circ} 1$, dans le coffre $\mathrm{A}$; à noter que les Gestes font de ce compromis un triomphe de l'évêque: Three Cartularies, p. 25 ; Les Gestes, t. 2, p. 100-101 - marquent le début d'une conservation organisée des archives de l'évêché (p. 7). Cela ne signifie pas l'absence de documents antérieurs: aux $\mathrm{IX}^{\mathrm{e}}$-XII ${ }^{\mathrm{e}}$ siècles, les rédacteurs des Gestes disposent de documents d'archives, en recopient quelques-uns, et signalent en particulier les legs des prélats à leur siège et leurs acquisitions en sa faveur. Selon la base de données Chartes originales antérieures à 1121 conservées en France, les plus anciens originaux connus en faveur de l'évêché sont deux diplômes de Charles le Simple, de 900 et 902 - Auxerre, AD Yonne, G1817; http://www.cn-telma.fr/originaux/charte723/ et ...charte724/ [dernier accès le 20 mars 2014]. Mais l'épiscopat du Clunisien Hugues de Mâcon (1136-1151) voit un très net durcissement de la politique épiscopale vis-à-vis du comte Les Gestes des évêques, t. 2, p. 20-22 : tournant politique majeur à Auxerre, qui provoque un changement dans l'attitude des évêques vis-à-vis de leurs droits et de leurs archives, et qui coïncide aussi, on l'a vu, avec une maturation décisive de l'idée qu'ils se font de leur histoire : dans l'état actuel du manuscrit 142, la notice d'Hugues constitue le point 
d'aboutissement de tout le texte précédent, et donc de toute la série épiscopale depuis les origines, dans une totale unité codicologique. Le fait que les plus anciens documents sont répartis dans des coffres différents suggère que ce classement en coffres est adopté sinon dès le départ, du moins assez tôt. On compte de deux à seize documents par décennie, les plus fournies étant les décennies 1200, 1210 et 1240 (douze à seize actes) : apparente intensification de l'activité juridique et administrative, qui coïncide avec «le temps des évêques princes » (Les Gestes, t. 2, p. 22).

Les deux autres cartulaires sont bien plus réduits. Celui du chapitre est en fait une épave, un bifolio, dont les premier et dernier actes sont mutilés. Le cartulaire du monastère féminin Saint-Julien est, lui, un dossier consacré à une unique affaire, un conflit contre les comtes de Joigny. Il réunit, dans l'ordre chronologique, l'acte de fondation par l'évêque Pallade - 631 ; p. 187, l'éditrice l'estime authentique -, deux privilèges $\mathrm{du} \mathrm{Ix}^{\mathrm{e}}$ siècle et les onze documents des $\mathrm{XI}^{\mathrm{e}}$-XIII ${ }^{\mathrm{e}}$ siècles relatifs à ce conflit, intégralement transcrits avec leurs très nombreuses répétitions. Pour chacun des trois cartulaires, l'éditrice respecte l'ordre du manuscrit; chaque pièce est précédée d'une analyse et suivie d'un court commentaire, en particulier s'il faut en discuter la datation. Signalons que trois actes du cartulaire de l'évêché, les numéros 14, 58 et 69 (années 1267-1275) sont en français.

siècle apparaît au total à Auxerre comme le grand moment de mise en ordre de la mémoire par l'écriture : "neuvième continuation » des Gestes épiscopaux - on a signalé son exceptionnelle précision - peu après 1223 , compilation du cartulaire du chapitre dans les années 1230, nouvelle continuation des Gestes peu après 1247, compilation du Grand Cartulaire de Saint-Germain par Gui entre 1266 et 1285, nouvelles continuations des Gestes peu après 1270 et 1278 respectivement, compilation du cartulaire de l'évêché dans les années 1280, achèvement des Gestes abbatiaux en 1290, compilation du Petit Cartulaire de Saint-Germain - AUXERRE, BM, ms. 162G - et du cartulaire de Saint-Julien dans les années 1290. C'est quasi-simultanément, dans les deux dernières décennies du siècle, que les deux grandes églises auxerroises, la cathédrale et Saint-Germain, font un effort particulier de classement de leurs archives et d'écriture - ou de poursuite de l'écriture - de leur histoire. L'historiographie médiévale auxerroise présente donc à la fois une forte continuité et un caractère nettement archaïsant : le genre, d'origine carolingienne, des Gestes, est irrégulièrement mais très longtemps réactivé par les chanoines ; c'est de façon plutôt anachronique que l'abbé y recourt à son tour à la fin du XIII ${ }^{e}$ siècle, mais justement à l'imitation du chapitre voisin.

17 À la page 39 du tome 3 des Gestes des évêques, les éditeurs notent que «l'histoire de l'église d'Auxerre au XIII e siècle reste à faire "; Constance Bouchard fait exactement le même constat à la page 3 de sa propre introduction. La publication rapprochée des cinq volumes que l'on vient de présenter met désormais à portée des chercheurs une partie non négligeable des sources - les deux cartulaires de Saint-Germain demeurent totalement inédits, la base de données Chartae Burgundiae Medii Aevi ne signalant pour Auxerre que les trois recueils ici décrits: http://www.artehis-cnrs.fr/pagedocumentaire-CBMA [dernier accès le 20 mars 2014] - et permettra, on le souhaite, de commencer à combler cette lacune.

Reçu : 25 mars 2014 - Accepté : 10 avril 2014. 
INDEX

Index géographique : France/Auxerre

Mots-clés : diplomatique, historiographie

\section{AUTEUR}

THOMAS GRANIER

Université Paul-Valéry Montpellier 3 